\title{
The 4-Point $\alpha$-Ary Approximating Subdivision Scheme
}

\author{
Abdul Ghaffar ${ }^{1}$, Ghulam Mustafa ${ }^{1}$, Kaihuai Qin ${ }^{2}$ \\ ${ }^{1}$ Department of Mathematics, The Islamia University of Bahawalpur, Bahawalpur, Pakistan \\ ${ }^{2}$ Dept. of Computer Science \& Technology, Tsinghua University, Beijing 100084, P. R. China \\ Email: abdulghaffar.jaffar@gmail.com, ghulam.mustafa@iub.edu.pk, qkh-dcs@tsinghua.edu.cn
}

Received 2013

\begin{abstract}
A general formula for 4-point $\alpha$-Ary approximating subdivision scheme for curve designing is introduced for any arity $\quad \alpha \geq 2$. The new scheme is extension of B-spline of degree 6 . Laurent polynomial method is used to investigate the continuity of the scheme. The variety of effects can be achieved in correspondence for different values of parameter. The applications of the proposed scheme are illustrated in comparison with the established subdivision schemes.
\end{abstract}

Keywords: Approximating Subdivision Scheme; Binary; Ternary; $\alpha$-Ary; Continuity; Convergence and Shape Parameters

\section{Introduction}

Subdivision modeling methods are effective algorithms to generate continuous curves and surfaces from a discrete set of control points by subdividing them according to some refining rules, recursively. Repetition of this process produces a very good approximation to the curve or surface defined by the original set of control points. In recent years, the subject of subdivision gained popularity due to some new applications, such as in the $3 \mathrm{D}$ animation industry. The next venture is to introduce these methods to be more consistent and efficient to the world of geometric modeling in the industry.

Approximating schemes were first developed by Rham and Chaikin [1,2]. Consequent to this, a lot of work has been done by different authors in the field of binary subdivision schemes, but the research communities are interested in introducing higher arity schemes (i.e. ternary, quaternary, ...n-ary) which give better result and less computational cost.

In late 80,s with the help of wavelet theory, a relationship between subdivision scheme and the "mask" of refinable function have been developed. Lian $[3,4]$ has introduced 3, 4, 5 and 6-point a-ary interpolating schemes. In these research papers, Lian used wavelets theory, a relatively new subject area that has been deeply studied for the last two decades or so, and found many successful applications. Lian [5] also offered $2 m$ and ( $2 m$ $+1)$-point interpolating a-ary schemes for curve designing. In 2009, Mustafa and Khan [6] for the first time introduced a new 4-point quaternary approximating subdivision scheme. Mustafa and Najma [7] presented same perspective for constructing $(2 b+2)$ and $(2 b+4)$-point $n$-ary interpolating and approximating schemes. Ghaffar et al. presented unified 3-point $\alpha$-ary approximation schemes and discussed various properties [8]. This motivates us to present 4-point $\alpha$-ary approximating scheme with high smoothness and more degree of freedom for curve designing. One of the main objectives of current work is to extend "The B-Spline of degree 6" to 4-point $\alpha$-ary approximating subdivision scheme.

This paper is organized as follows: General form of 4-point $\alpha$-ary subdivision scheme is constructed in Section 2. The family of 4-point $\alpha$-ary approximating scheme, basic properties and analysis are presented in Section 3. Comparison of 4-point $\alpha$-ary schemes is given in Section 4. Finally conclusion is given in Section 5 .

\section{Main Results}

In this section, we are introducing family of 4-point $\alpha$-ary approximating subdivision scheme for curve designing for any arity $\alpha \geq 2$. This family is the extension of B-spline of degree six i.e. (4-point approximating subdivision scheme):

$$
\left\{\begin{array}{l}
f_{2 i}^{k+1}=\frac{7}{64} f_{i-1}^{k}+\frac{35}{64} f_{i}^{k}+\frac{21}{64} f_{i+1}^{k}+\frac{1}{64} f_{i+2}^{k}, \\
f_{2 i+1}^{k+1}=\frac{1}{64} f_{i-1}^{k}+\frac{21}{64} f_{i}^{k}+\frac{35}{64} f_{i+1}^{k}+\frac{7}{64} f_{i+2}^{k} .
\end{array}\right.
$$

If there exist a unique $l^{2}$-sequence $\left\{P_{k}\right\}$ that describes the "two scale equation"

$$
\phi(x)=\sum_{k=-\infty}^{\infty} P_{k} \phi(2 x-k),
$$


of scaling function $\phi$. Then corresponding to this $l^{2}$ sequence, let us introduce the notation

$$
P(z)=P_{\Phi}(z)=\frac{1}{2} \sum_{k=-\infty}^{\infty} P_{k} z^{k},
$$

Due to the development of the wavelet theory, the schemes (1) can be easily re-discovered by the scaling functions $\phi_{4}$ satisfying the two-scale equations

$$
\begin{aligned}
\Phi_{4}(t)=\frac{1}{64} & \left\{\Phi_{4}(2 t)+7 \Phi_{4}(2 t-1)+21 \Phi_{4}(2 t-2)\right. \\
& +35 \Phi_{4}(2 t-3)+35 \Phi_{4}(2 t-4)+21 \Phi_{4}(2 t-5) \\
& \left.+7 \Phi_{4}(2 t-6)+\Phi_{4}(2 t-7)\right\}, t \epsilon R
\end{aligned}
$$

By taking the Fourier transforms of (4), we arrive at the following two-scale equations of $\phi_{4}$

$$
\begin{aligned}
\hat{\Phi}_{4}(w)=\frac{1}{64} & \left\{\frac{1}{2} \hat{\Phi}_{4}\left(\frac{w}{2}\right)+\frac{7}{2} e^{\frac{i w}{2}} \hat{\Phi}_{4}\left(\frac{w}{2}\right)+\frac{21}{2} e^{i w} \hat{\Phi}_{4}\left(\frac{w}{2}\right)\right. \\
& +\frac{35}{2} e^{\frac{3 i w}{2}} \hat{\Phi}_{4}\left(\frac{w}{2}\right)+\frac{35}{2} e^{2 i w} \hat{\Phi}_{4}\left(\frac{w}{2}\right) \\
& +\frac{21}{2} e^{\frac{5 i w}{2}} \hat{\Phi}_{4}\left(\frac{w}{2}\right)+\frac{7}{2} e^{3 i w} \hat{\Phi}_{4}\left(\frac{w}{2}\right) \\
& \left.+\frac{1}{2} e^{\frac{7 i w}{2}} \hat{\Phi}_{4}\left(\frac{w}{2}\right)\right\} .
\end{aligned}
$$

This implies

$$
\hat{\Phi}_{4}(w)=\frac{1}{2} \hat{\Phi}_{4}\left(\frac{w}{2}\right) \frac{1}{(4)^{3}}\left(\frac{1-\left(e^{\frac{i w}{2}}\right)^{2}}{1-e^{\frac{i w}{2}}}\right)^{4} \sum_{j=0}^{3}\left(\begin{array}{l}
3 \\
j
\end{array}\right)\left(e^{\frac{i w}{2}}\right)^{j} .
$$

This normalization simplifies to the following Fourier transform formulation:

$$
\hat{\Phi}_{4}(w)=\frac{1}{2} \hat{\Phi}_{4}\left(\frac{w}{2}\right) P_{4}(z),
$$

where

$$
P_{4}(z)=\frac{1}{(4)^{3}}\left(\frac{1-z^{2}}{1-z}\right)^{4} \sum_{j=0}^{3}\left(\begin{array}{l}
3 \\
j
\end{array}\right) z^{j},
$$

and $z=e^{\frac{i w}{2}}$.

By introducing the shape parameter $u_{j}$ in (5), we get

$$
P_{4}(z)=\frac{1}{(4)^{2}}\left(\frac{1-z^{2}}{1-z}\right)^{4} \sum_{j=0}^{3} \frac{1}{4}\left(\begin{array}{l}
3 \\
j
\end{array}\right) u_{j} z^{j} .
$$

By taking $u_{0}=u_{3}=4 w$ and $u_{1}=u_{2}=4(1-w)$, we get

$$
P_{4}(z)=\frac{1}{16}\left(\frac{\left(1-z^{2}\right)}{(1-z)}\right)^{4} w+(1-w) z+(1-w) z^{2}+w z^{3} .
$$

Now, if we allow the scaling factor, denoted by $a$, to be $\geq 2$, and denote such a scaling function denoted by ${ }^{a} \phi$, then the Fourier transforms of two-scale equation of ${ }^{a} \phi$ becomes

$$
\widehat{{ }^{a} \phi}(w)=\widehat{{ }^{a} \phi}\left(\frac{w}{a}\right){ }^{a} P\left(e^{\frac{i w}{a}}\right),
$$

and

$$
\widehat{{ }^{a} \phi}(w)=\widehat{a} \phi\left(\frac{w}{a}\right){ }^{a} P(z) .
$$

where $z=e^{(i w / a)}$ and again, ${ }^{a} P$ is its two-scale symbol and ${ }^{a} \phi$ is then equivalent to ${ }^{a} P$ satisfies

$$
P_{4}^{a}(Z)=\frac{1}{\left(2 a^{3}\right)}\left(\frac{\left(1-z^{a}\right)}{(1-z)}\right)^{4} w+(1-w) z+(1-w) z^{2}+w z^{3} .
$$

\section{Family of 4-Point $a$-Ary Schemes}

In this section, we discuss only three 4-point schemes. For setting $a=2,3$ and 4 in (7), we obtain three polynomials $P_{4}^{a}(z), a=2,3,4$ with following sets of coefficients called the mask of the 4-point binary, ternary and quaternary schemes, respectively.

$$
\begin{aligned}
& \alpha_{4}^{2}=\frac{1}{16}\left\{\begin{array}{c}
w, 1+3 w, 5+w, 10-5 w, \\
10-5 w, 5+w, 1+3 w, w
\end{array}\right\}, \\
& \alpha_{4}^{3}=\frac{1}{54}\left\{\begin{array}{c}
w, 1+3 w, 5+5 w, 14+3 w, 26-3 w \\
35-9 w, 35-9 w, 26-3 w, 14+3 w, \\
5+5 w, 1+3 w, w
\end{array}\right\},
\end{aligned}
$$

and

$$
\alpha_{4}^{4}=\frac{1}{128}\left\{\begin{array}{c}
w, 1+3 w, 5+5 w, 14+7 w, 30+5 w \\
51-w, 71-7 w, 84-13 w, 84-13 w \\
71-7 w, 51-w, 30+5 w, 14+7 w \\
5+5 w, 1+3 w, w
\end{array}\right\},
$$

Order of continuities of the above schemes is given in Table 1. One can easily find the order of continuity over the parametric intervals by using the approach of [9].

\subsection{Support of Basic Limit Function}

The basic function of a subdivision scheme is the limit function of the proposed scheme for the following data

$$
f_{i}^{0}=\left\{\begin{array}{l}
1, i=0 \\
0, i \neq 0
\end{array}\right.
$$

The following theorem is related to the support of basic limit functions of the 4-point $a$-ary scheme.

Theorem 1. The basic limit functions of ${ }^{a} \Phi_{4}$ proposed 4-point a-ary approximating schemes have support 
Table 1. The order of continuity of proposed 4-point $a$-ary schemes for certain ranges of parameter.

\begin{tabular}{cccccc}
\hline Scheme & Parameter & $C^{n}$ & Scheme & Parameter & $C^{n}$ \\
\hline Binary & $-\frac{3}{2}<w<\frac{5}{2}$ & $C^{0}$ & Quaternary & $-14<w<\frac{33}{2}$ & $C^{0}$ \\
$\ldots$ & $-\frac{3}{2}<w<\frac{5}{2}$ & $C^{1}$ & $\ldots$ & $-\frac{13}{2}<w<\frac{19}{2}$ & $C^{1}$ \\
$\ldots$ & $-\frac{1}{2}<w<\frac{3}{2}$ & $C^{2}$ & $\ldots$ & $-3<w<5$ & $C^{2}$ \\
$\ldots$ & $-\frac{1}{2}<w<\frac{3}{2}$ & $C^{3}$ & $\ldots$ & $-1<w<3$ & $C^{3}$ \\
$\ldots$ & $0<w<1$ & & \\
Ternary & $-\frac{23}{4}<w<\frac{31}{4}$ & $C^{4}$ & & & \\
$\ldots$ & $-\frac{7}{2}<w<\frac{11}{2}$ & $C^{1}$ & & & \\
$\ldots$ & $-2<w<4$ & $-1<w<3$ & $C^{2}$ & & \\
\hline
\end{tabular}

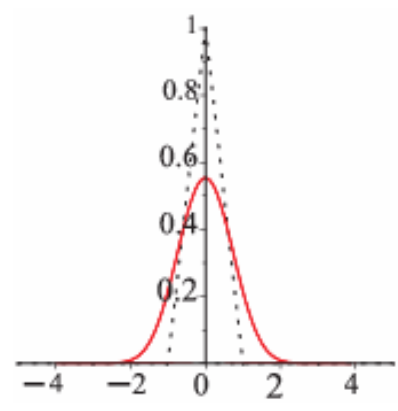

(a)

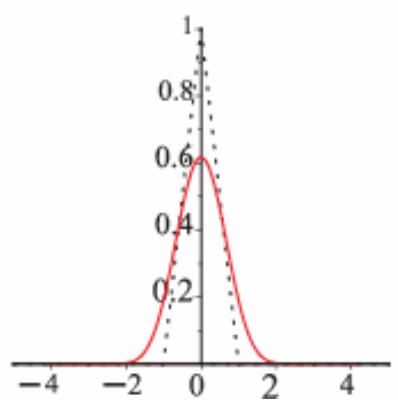

(b)

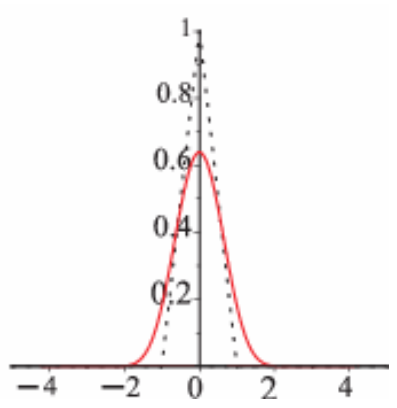

(c)

Figure 1. (a-c) represent the basic limit functions of the 4-point binary, ternary and quaternary schemes, respectively.

width $s=\frac{4 a-1}{a-1}$, for $a=2,3,4, \ldots, m$, which implies that it vanishes outside the interval $\left[-\frac{4 a-1}{2(a-1)}, \frac{4 a-1}{2(a-1)}\right]$.

Proof. Since the basic function is the limit function of the scheme corresponding to (7) for the data (11), its support width "s" can be determine by computing how far the effect of the non-zero vertex $f_{0}^{0}$ will propagate along by. As the mask of $a$-ary 4-point scheme is $4 a-1$ long sequence by centering it on that vertex; the distances to the last of its left and right non-zero coefficients are equal to $\frac{4 a-1}{2}$ At the first subdivision step we see that the vertices on the both sides of $f_{0}^{1}$ at $\frac{4 a-1}{2}$ are the furthest non-zero new vertices. At each refinement, the distances on both sides are reduced by the factor $\frac{1}{a}$. At the next step of the scheme, this will propagate along by $\frac{4 a-1}{2} \times \frac{1}{a}$ on both sides. Hence after $k$ subdivision steps the furthest non-zero vertex on the left will be at

$$
\begin{aligned}
& \left(\frac{4 a-1}{2}\right)\left(\frac{1}{a}+\frac{1}{a^{2}}+\ldots+\frac{1}{a^{k}}\right) \\
= & \left(\frac{4 a-1}{a}\right)\left(\sum_{j=0}^{k-1} \frac{1}{a^{j}}\right) .
\end{aligned}
$$

So the total support width is

$$
\text { (2) }\left(\frac{4 a-1}{a}\right)\left(\sum_{j=0}^{\infty} \frac{1}{a^{j}}\right)=\frac{4 a-1}{a-1} \text {. }
$$

\subsection{Precision Set}

For approximating schemes, we do not expect new vertices to be lie on the same curve as old ones, so it is necessary to look to see whether all the vertices lie on a common curve. We can calculate the order of precision by using the technique as given in [10]. 
Lemma 2. The scheme (3.1) has cubic precision.

Proof. We carry out this result by taking our origin the middle of an original span with ordinate

$$
\ldots,(-5)^{n},(-3)^{n},(-1)^{n},(1)^{n},(3)^{n},(5)^{n}, \ldots
$$

If $y=x^{n}$, then we have

$$
\begin{gathered}
{[y]=\ldots, a_{1}(-5)^{n}+a_{2}(-3)^{n}+a_{3}(-1)^{n}+a_{4}(1)^{n},} \\
a_{4}(-5)^{n}+a_{3}(-3)^{n}+a_{2}(-1)^{n}+a_{1}(1)^{n}, \\
a_{1}(-3)^{n}+a_{1}(-1)^{n}+a_{1}(1)^{n}+a_{1}(3)^{n}, \\
a_{4}(-3)^{n}+a_{3}(-1)^{n}+a_{2}(1)^{n}+a_{1}(3)^{n}, \\
\vdots, \\
\\
a_{4}(-1)^{n}+a_{3}(1)^{n}+a_{2}(3)^{n}+a_{1}(5)^{n},
\end{gathered}
$$

where

$$
a_{1}=\frac{3}{16}, a_{2}=\frac{7}{16}, a_{3}=\frac{5}{16}, a_{4}=\frac{1}{16} .
$$

If $y=x^{1}$, then we have

$$
\begin{aligned}
& {[y]=\ldots,-\frac{5}{2},-\frac{3}{2},-\frac{1}{2}, \frac{1}{2}, \frac{3}{2}, \frac{5}{2}, \ldots} \\
& {[\delta y]=\ldots, 1,1,1,1,1, \ldots} \\
& {\left[\delta^{2} y\right]=0,}
\end{aligned}
$$

where $\delta$ represents the differences of the vertices.

If $y=x^{2}$, then we have

$$
\begin{gathered}
{[y]=\ldots, \frac{15}{2}+2 w, \frac{7}{2}+2 w, \frac{3}{2}+2 w, \frac{3}{2}+2 w,} \\
\frac{7}{2}+2 w, \frac{15}{2}+2 w, \ldots
\end{gathered}
$$

Taking further differences, we get $\left[\delta^{3} y\right]=0$. If $y=x^{3}$, then we have

$$
\begin{gathered}
{[y]=\ldots,-25-15 w,-9-9 w,-2-2 w,} \\
2+2 w, 9+9 w, 25+15 w, \ldots
\end{gathered}
$$

by taking further differences, we get $t\left[\delta^{4} \mathrm{y}\right]=0$.

Thus the scheme has cubic precision.

For this analysis we observe that the scheme (8) is designed so that it has cubic precision for any value of $w$. While the schemes (9) and (10) have cubic precision for any value of $\mathrm{w}$ and with the special values $\mathrm{w}=\frac{1}{3}$ and $\mathrm{w}=\frac{1}{2}$, the schemes have quartic precision, respectively.

\section{Comparison and Application}

In this section, we show that the popular existing schemes are special cases of our proposed family of schemes (8)(10).

- With the special values of parameter ( $\mathrm{w}=0$ and $\mathrm{w}=\frac{1}{4}$ ), the subdivision schemes generated by B-splines of degree 4 and 6 , respectively, are obtained from the schemes (8).

- Moreover, the proposed scheme (8) is a generalized scheme, as the mask of the proposed scheme coincides with the mask of schemes [9,11-13] after setting $w=-\frac{5}{8}$, $\mathrm{w}=0, \mathrm{w}=\frac{1}{6}+\omega$ and $w=u$ respectively.

- It may be noted that if we put

$$
\mathrm{w}=\frac{1}{6}+\omega, \mathrm{w}=-\frac{1}{162}+\frac{1}{3} \mathrm{u}
$$

and

$$
\mathrm{w}=-\frac{35}{24}
$$

in (9), the proposed 4-point scheme can also be considered as the general form of the stationary 4-point ternary approximating schemes of $[8,13,16]$, respectively.

- For substituting $\mathrm{w}=-\frac{21}{8}$, the obtained mask of scheme (10) coincides with mask of the famous 4-point quaternary scheme of Ko [17].

Here we compare our proposed schemes with the existing binary, ternary and quaternary schemes (see Table 2). It is observed that the continuity and approximation order of proposed schemes are better than the existing schemes. Moreover, Figure 2 is exposed to show the role of free parameters when the schemes (8)-(10) applied on discrete data points. In Figure 2(a), black, red and blue lines show the visual smoothness of the binary scheme at the parametric values $w=0, w=\frac{1}{8}$ and $w=\frac{1}{2}$, respectively. In Figure 2(b), blue, green, black and red lines show the visual smoothness of the ternary scheme at the parametric values $w=-1, w=-\frac{1}{2}, w=0$, and $w=\frac{1}{2}$, respectively. In Figure 2(c), blue, green, black and red lines show the visual smoothness of the quaternary scheme at the parametric values $w=-1$, $w=-\frac{1}{2}, w=0$ and $w=\frac{1}{2}$, respectively. From Figure 2, we conclude that the behavior of the limiting curve acts as tightness/looseness when the values of free parameter vary. 
Table 2. Comparison of 4-point ternary schemes.

\begin{tabular}{llccc}
\hline \multicolumn{1}{c}{ Scheme } & Type & Support & Order & $\boldsymbol{C}^{n}$ \\
\hline Binary 4-point [18] & Interpolating & 6 & 4 & 1 \\
\hline Binary 4-point [19] & Interpolating & 6 & 4 & 1 \\
Binary 4-point [12] & Approximating & 7 & 4 & 2 \\
\hline Ternary 4-point [20] & Interpolating & 5 & 4 & 1 \\
\hline Ternary 4-point [21, 3] & Interpolating & 5 & 3 & 1 \\
Ternary 4-point [22] & terpolatingIn & 5 & 3 & 2 \\
\hline Ternary 4-point [3] & Interpolating & 5 & 3 & 1 \\
Ternary 4-point [16] & Approximating & 5.5 & 4 & 2 \\
\hline Quaternary 4-point [17] & Approximating & 5 & 4 & 2 \\
Quaternary 4-point [6] & Approximating & 5 & 4 & 3 \\
Binary 4-point proposed & Approximating & 7 & 3 & 5 \\
Ternary 4-point proposed & Approximating & 5.5 & 5 & 3 \\
\hline Quaternary 4-point proposed & Approximating & 5 & 5 & 3 \\
\hline
\end{tabular}

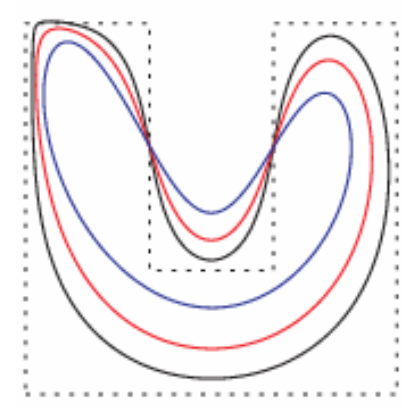

(a)

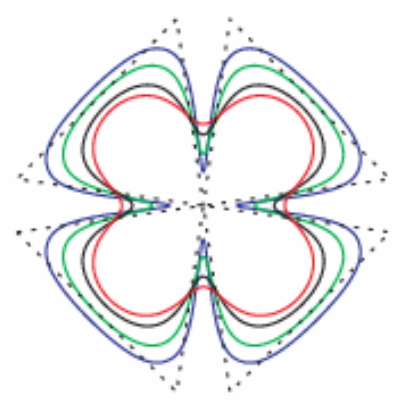

(b)

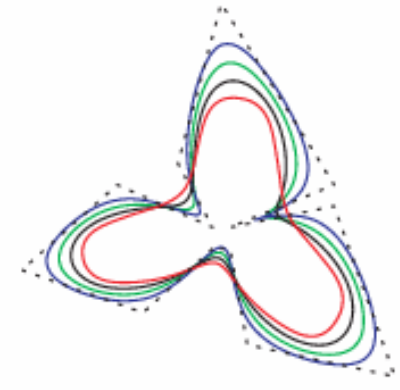

(c)

Figure 2. (a-c) show the role of shape parameter of the schemes (8), (9) and (10) respectively.

\section{Conclusions}

The families of 4-point a-ary approximating schemes for curve design were established in general. The first family is binary, the second is ternary and the third family is quaternary approximating. The support of subdivision scheme influences the locality. One of the best ways to get a smaller support is to raise the arity. This is one of the advantages of the proposal of the scheme. We have also studied its continuity and determined its order of precision. It is observed that the order of precision set increases by increasing the arity of the schemes while the continuity decreases by increasing the arity of the scheme. Moreover, we have showed that several previously proposed schemes are members of this family. It has been shown that proposed schemes are better than existing schemes in the sense of smoothness and approximation order.

\section{Acknowledgement}

This work is supported by the Beijing Municipal Natural Science Foundation (4102027), the National Natural Science Foundation of China (60973101) and HEC Pakistan.

\section{REFERENCES}

[1] G. de Rham, "Un Peude Mathematiques a Proposed Une Courbe Plane," Revwede Mathematiques Elementry II, Oevred Completes, 1947, pp. 678-689.

[2] G. M. Chaikin, "An Algorithm for High-Speed Curve Generation," Computer Graphics and Image Processing, Vol. 3, No. 4, 1974, pp. 346-349. doi:10.1016/0146-664X(74)90028-8

[3] J. -A. Lian, "On A-ary Subdivision for Curve Design: I. 4-point and 6-point Interpolatory Schemes," Applications 
and Applied Mathematics: An International Journal, Vol. 3, No. 1, 2008, pp.18-29.

[4] J. -A. Lian, "On A-ary Subdivision for Curve Design: II. 3-point and 5-point Interpolatory Schemes," Applications and Applied Mathematics: An International Journal, Vol. 3, No. 2, 2008, pp. 176-187.

[5] J. -A. Lian, "On a-ary Subdivision for Curve design: III. $2 \mathrm{~m}$-point and $(2 \mathrm{~m}+1)$ point Interpolatory Schemes," Applications and Applied Mathematics: An International Journal, Vol. 4, No. 2, 2009, pp. 434-444.

[6] G. Mustafa and F. Khan, "A New 4-point $c^{\text {x }}$ Quaternary Approximating Subdivision Scheme," Abstract and Applied Analysis, Vol. 2009, Article ID 301967, 14 pages.

[7] G. Mustafa and A. R. Najma , "The Mask of $(2 b+$ 4)-point n-ary Subdivision Scheme," Computing, Vol. 90, No. 1-2, 2010, pp. 1-14. doi:10.1007/s00607-010-0108-x

[8] A. Ghaffar, G. Mustafa and K. Qin, "Unification and Application of 3-point Approximating Subdivision Schemes of Varying Arity," Open Journal of Applied Sciences, Vol. 2, No. 4B, 2012, pp. 48-52. doi:10.4236/ojapps.2012.24B012

[9] M. F. Hassan and N. A. Dodgson, "Ternary and Three-point Univariate Subdivision Schemes," In: A. Cohen, J. L. Marrien, L. L. Schumaker (Eds.), Curve and Surface Fitting: Sant-Malo2002, Nashboro Press, Brentwood, 2003, pp. 199-208.

[10] N. Dyn, “Analysis of Convergence and Smoothness by the Formalism of Laurent Polynomials," In: A.Iske, E. Quak, M. S Floater (Eds), Tutorials on Multiresolution in Geometric Modelling, Springer, 2002, pp. 51-68. doi:10.1007/978-3-662-04388-2 3

[11] G. Mustafa, F. Khan and A. Ghaffar, "The m-Point Approximating Subdivision Scheme," Lobachevskii Journal of Mathematics, Vol. 30, No. 2, 2009, pp. 138-145. doi:10.1134/S1995080209020061

[12] N. Dyn, M. S. Floater and K. Horman, "A $c^{2}$ Four-Point Subdivision Scheme with Fourth Order Accuracy and its Extension," In Mathematical Methods for Curves and Surfaces: Tromso 2004, M. Daehlen, K. Morken, and L.
L. Schumaker (eds.), 2005, pp. 145-156.

[13] H. Zheng, M. Hu and G. Peng, "P-ary Subdivision Generalizing B-splines," Second International Conference: On Computer and Electrical Engineering, 2009, pp. 214-218. doi:10.1109/ICCEE.2009.204

[14] A. Ghaffar and G. Mustafa, "A Family of Even-Point ternary Approximating Schemes," ISRN Applied Mathematics, Vol. 2012, 2012, Article ID 197383, 14 pages.

[15] H. Zheng, M. Hu and G. Peng, "Ternary Even Symmetric 2n-point Subdivision," International Conference on: Computational Intelligence and Software Engineering,2009, pp. 1-4.doi:10.1109/CISE.2009.5363033

[16] K. P. Ko, B. -G. Lee and G. Joon Yoon. "A Ternary 4-point Approximating Subdivision Scheme," Applied Mathematics and Computation, Vol. 190, 2007

[17] K. P. Ko, "A Quatnary Approximating 4-point Subdivision Scheme," J. KSIAM, Vol. 13, No. 4, 2009, PP. 307-341.

[18] C. Beccari, G. Casciola and L. Romani, "A Non-stationary Uniform Tension Controlled Interpolating 4-point Scheme Reproducing Conics," Computer Aided Geometric Design, Vol. 24, No. 1, 2007, pp. 1-9.

[19] G. Casciola and L. Romani. "An Interpolating 4-point $c^{\text {" }}$ Ternarynon-stationary Subdivision Scheme with Tension Control," Computer Aided Geometric Design, Vol. 24, No. 2, 2007, pp. 210-219.

[20] G. Casciola and L. Romani, "Shape Controlled Interpolatory Ternary Subdivision," Applied Mathematics and Computation, Vol. 215, No. 1, 2009, pp. 916-927.

[21] G. Deslauriers and S. Dubic, "Symmetric Iterative Interpolation Process," Constractive Approximation, Vol. 5, No. 1, 1989, pp. 49-68. doi:10.1007/BF01889598

[22] M. F. Hassan, I. P. Ivrissimitzis, N. A. Dodgson and M. A. Sabin, "An Interpolating 4-points $C^{2}$ Ternary Stationary Subdivision Scheme," Computer Aided Geometric Design, Vol. 19, 2002, pp. 1-18. 